\title{
The Relation of Solutions Between Different Models of Support Vector Regression
}

\author{
Meiqin Pan \\ School of Business and Management, Shanghai International Studies University Shanghai, China
}

\begin{abstract}
The relations of solutions between different models of Support Vector Regression are proposed in this paper. Usually, Support Vector Regression (SVR) is formulated as a convex quadratic programming with bound constrains. With different improvements, different improved regression models and their strong convex programming have come into being. Basing on Lagrange function and KKT conditions, this paper proves strictly that the solution of improved model is the solution of prime model. Which provides the SVR with theory base.
\end{abstract}

Keywords-quadratic rogramming; lagrange function; kkt condition

\section{INTRODUCTION}

As an important method of Pattern Recognition, Support Vector Machine (SVM) is widely used in machine learning areas, especially in classification problem. It is a completely new kind of machine learning algorithm proposed by Vapnik and his collaborators [1], and is based on the structural risk minimization principle in statistical learning theory. By combining the classification idea of maximum separation plane with the kernel-based method, SVM shows an excellent generalization capability[2,4,6,8]. With the deeper and deeper studying, more and more improved algorithms[10,11] have been proposed. Especially, Support Vector Regression (SVR) is proposed as a new interest field of Support Vector Machine (SVM).

Conventionally, SVR is formulated as a constrained minimization problem, named a convex quadratic programming problem, which maximize the margin between the parallel separating planes by optimizing with respect to $\mathrm{w}$ (normal to the regression plane) for a fixed b(location relative to the origin of regression plane).With different models and different techniques, different regression algorithms have come into being. SOR-SVR[3] modifies the primal programming problem, that is, maximizes the margin between the parallel separating planes by optimizing with respect to both $\mathrm{w}$ and $\mathrm{b}$, which induces the problem strong convexity. With the SOR algorithm and the gradient optimality condition, the iteration of SOR-SVR is given out. Active-SVR[5] , Lagrange-SVR[7] and Smooth-SVR[9] are based on the same modification, the distance between parallel separating planes is measured in the $(n+1)$-dimensional space of $(w, b)$, the square of 2-norm of the

slack variable $\bar{\xi}$ and $\hat{\xi}$ is minimized with weigh $C / 2$ instead of the 1 -norm of $\bar{\xi}$ and $\hat{\xi}$. These facts make the constraint $\bar{\xi} \geq 0$ and $\hat{\xi} \geq 0$ redundant and induces the problem strong convexity, simplifies the problem.

However, in above algorithms, the relation of solutions between improved problem and primal problem is not provided, they just cite the result of SOR-SVM[2]. Since the classification and regression have different models, this citation does not reasonable. This paper bases on the form of improved problem and primal problem, relates with the relation of solutions between improved problem and primal problem of SVM, studies the property of solutions between improved problem and primal problem of SVR, and proves this property strictly. Which provides the SVR with theory bases.

\section{SUPPORT VECTOR REGRESSION}

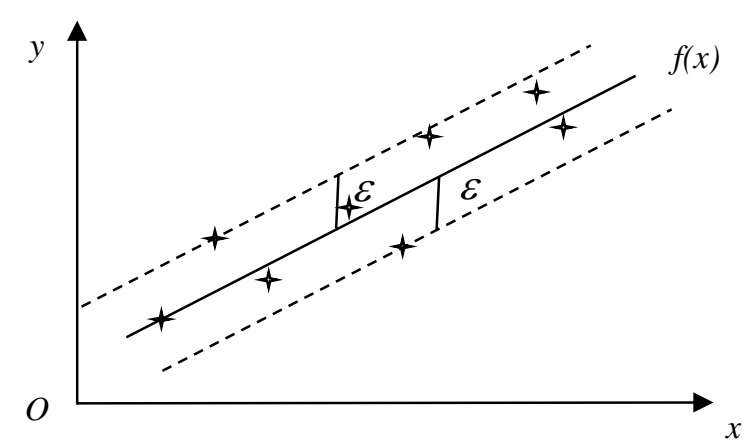

THE LINEAR REGRESSION PROBLEM OF TWO-DIMENSION

In regression problems, suppose $X_{i} \in R^{n}$ is an input data and $y_{i} \in R$ is a target output. We are given a training data set $S=\left\{\left(x_{1}, y_{1}\right), \ldots,\left(x_{m}, y_{m}\right)\right\} \subseteq R^{n} \times R$, The task of SVR is to find a function $f(x)$ that can correctly predict the observation values $y$, of the new input points $x$, by learning from the given training data set $\mathrm{S}$ (Fig 1). Here, learning from a given training data set means finding a linear or nonlinear hyperplane that tolerates a small error in fitting this training data set. Disregarding the tiny errors that fall within some tolerance, say $\varepsilon$, that may lead to a better generalization ability is achieved by utilizing an $\varepsilon$-insensitive loss function. Also, applying the idea of SVM, the function $f(x)$ is made as flat as possible in fitting the training data. This problem is called $\varepsilon$-support vector regression ( $\varepsilon$-SVR) and a data point $x_{i} \in R^{n}$ is called a support vector if $f\left(x_{i}\right)-y_{i} \geq \varepsilon$.Usually, 
$\varepsilon$-SVR can be changed into SVM as following method. Adding $\varepsilon$ and decreasing $\varepsilon$ to y of the training point, we get two sets of positive points and minus points, which forms a classification problem about these two sets. So the hyperplane solving from SVM is the regression function of SVR.

The model of Support Vector Regression is as follows:

$$
\begin{aligned}
\min _{(w, b, \bar{\xi}, \hat{\xi})} & \frac{1}{2} w^{T} w+C\left(e^{T} \bar{\xi}+e^{T} \hat{\xi}\right) \\
\text { s.t. } & A w+b e-y \leq \varepsilon e+\bar{\xi} \\
& -A w-b e+y \leq \varepsilon e+\hat{\xi} \\
& \bar{\xi}, \hat{\xi} \geq 0
\end{aligned}
$$

Model (2.1) is formulated as a constrained and convex quadratic programming problem, which maximize the margin between the parallel separating planes by optimizing with respect to ${ }^{w}$ (normal to the regression plane) for a fixed $b$ (location relative to the origin of regression plane). If we modify the formula (2.1) a little, maximize the margin between the parallel separating planes by optimizing with respect to both $w$ and $b$, which induces the problem strong convexity as following model (2.2):

$$
\begin{aligned}
& \min _{(w, b, \bar{\xi}, \hat{\xi})} \frac{1}{2}\left(w^{T} w+b^{2}\right)+\frac{C}{2}\left(e^{T} \bar{\xi}+e^{T} \hat{\xi}\right) \\
& \text { s.t. } A w+b e-y \leq \varepsilon e+\bar{\xi} \\
& \quad-A w-b e+y \leq \varepsilon e+\hat{\xi} \\
& \quad \bar{\xi}, \hat{\xi} \geq 0
\end{aligned}
$$

\section{THE RELATION OF SOLUTIONS}

Theorem 1. Each solution $(\tilde{w}, \tilde{b}, \tilde{\xi}, \tilde{\hat{\xi}})$ of (2.2) is a solution of (2.1) for a possibly larger value of $C$ replaced by $C+\max \left\{e^{T} \tilde{\hat{v}}, e^{T} \tilde{\bar{v}}\right\}$ in (2.1), if exist $\tilde{\bar{v}} \geq 0$ and $\tilde{\hat{v}} \geq 0$, such that $A^{T}(\tilde{\hat{v}}-\tilde{\bar{v}})=0, e^{T}(\tilde{\hat{v}}-\tilde{\bar{v}})=-\tilde{b}$,

$$
\varepsilon e^{T}(\tilde{\hat{v}}+\tilde{\bar{v}})+\tilde{\hat{\xi}}^{T} \tilde{\hat{v}}+\tilde{\xi}^{T} \tilde{\bar{v}}-y^{T}(\tilde{\hat{v}}-\tilde{\bar{v}}) \leq \widetilde{b}^{2} .
$$

Proof: The Lagrange function of (2.2) is:

$$
\begin{aligned}
& \quad L(w, b, \bar{\xi}, \hat{\xi}, \bar{\alpha}, \hat{\alpha}, \bar{\beta}, \hat{\beta}) \\
& =\frac{1}{2} w^{T} w+\frac{1}{2} b^{2}+C e^{T} \bar{\xi}+C e^{T} \hat{\xi}+\bar{\alpha}^{T}(A w+b e-y-\varepsilon e-\bar{\xi}) \\
& \quad-\hat{\alpha}^{T}(A w+b e-y+\varepsilon e+\hat{\xi})-\bar{\beta}^{T} \bar{\xi}-\hat{\beta}^{T} \hat{\xi} \\
& \text { Where } \bar{\alpha}, \hat{\alpha}, \bar{\beta}, \widehat{\beta} \text { are Lagrange multipliers. Its KKT }
\end{aligned}
$$
conditions are as follows:

$$
\left\{\begin{array} { l } 
{ \nabla _ { w } L = w + A ^ { T } \overline { \alpha } - A ^ { T } \hat { \alpha } = 0 } \\
{ \nabla _ { b } L = b + e ^ { T } \overline { \alpha } - e ^ { T } \hat { \alpha } = 0 } \\
{ \nabla _ { \overline { \xi } } L = C e - \overline { \alpha } - \overline { \beta } = 0 } \\
{ \nabla _ { \hat { \xi } } L = C e - \hat { \alpha } - \hat { \beta } = 0 } \\
{ \overline { \alpha } ^ { T } ( A w + b e - y - \varepsilon e - \overline { \xi } ) = 0 } \\
{ \hat { \alpha } ^ { T } ( A w + b e - y + \varepsilon e + \hat { \xi } ) = 0 }
\end{array} \quad \Rightarrow \left\{\begin{array}{l}
w=A^{T}(\hat{\alpha}-\bar{\alpha}) \\
b=e^{T}(\hat{\alpha}-\bar{\alpha}) \\
0 \leq \bar{\alpha} \leq C e \\
0 \leq \hat{\alpha} \leq C e \\
\bar{\alpha}^{T}(A w+b e-y-\varepsilon e-\bar{\xi})=0 \\
\hat{\alpha}^{T}(A w+b e-y+\varepsilon e+\hat{\xi})=0
\end{array}\right.\right.
$$

Suppose $(\tilde{w}, \tilde{b}, \tilde{\bar{\xi}}, \tilde{\hat{\xi}})$ is the solution of (2.2), so it is the KKT point of (2.2), then we get KKT conditions as follows:

$$
\left\{\begin{array}{l}
\tilde{w}=A^{T}(\tilde{\hat{\alpha}}-\widetilde{\bar{\alpha}}) \\
\tilde{b}=e^{T}(\tilde{\hat{\alpha}}-\widetilde{\bar{\alpha}}) \\
0 \leq \widetilde{\bar{\alpha}} \leq C e \\
0 \leq \tilde{\hat{\alpha}} \leq C e \\
\widetilde{\alpha}^{T}(A \tilde{w}+\tilde{b} e-y-\varepsilon e-\tilde{\bar{\xi}})=0 \\
\tilde{\hat{\alpha}}^{T}(A \tilde{w}+\tilde{b} e-y+\varepsilon e+\tilde{\hat{\xi}})=0
\end{array}\right.
$$

Now, we validate $(\tilde{w}, \tilde{b}, \tilde{\xi}, \tilde{\hat{\xi}})$ is the KKT point of (2.1). The Lagrange function of (2.1) is:

$$
\begin{aligned}
& L(w, b, \bar{\xi}, \hat{\xi}, \bar{\alpha}, \hat{\alpha}, \bar{\beta}, \widehat{\beta}) \\
= & \frac{1}{2} w^{T} w+C e^{T} \bar{\xi}+C e^{T} \hat{\xi}+\bar{\alpha}^{T}(A w+b e-y-\varepsilon e-\bar{\xi}) \\
& -\hat{\alpha}^{T}(A w+b e-y+\varepsilon e+\hat{\xi})-\bar{\beta}^{T} \bar{\xi}-\widehat{\beta}^{T} \hat{\xi}
\end{aligned}
$$

Where $\bar{\alpha}, \hat{\alpha}, \bar{\beta}, \hat{\beta}$ are Lagrange multipliers. Its KKT conditions are as following:

$$
\begin{gathered}
\left\{\begin{array}{l}
\nabla_{w} L=w+A^{T} \bar{\alpha}-A^{T} \hat{\alpha}=0 \\
\nabla_{b} L=e^{T} \bar{\alpha}-e^{T} \hat{\alpha}=0 \\
\nabla_{\bar{\xi}} L=C e-\bar{\alpha}-\bar{\beta}=0 \\
\nabla_{\hat{\xi}} L=C e-\widehat{\alpha}-\widehat{\beta}=0 \\
\bar{\alpha}^{T}(A w+b e-y-\varepsilon e-\bar{\xi})=0 \\
\hat{\alpha}^{T}(A w+b e-y+\varepsilon e+\hat{\xi})=0
\end{array}\right. \\
\Rightarrow\left\{\begin{array}{l}
w=A^{T}(\hat{\alpha}-\bar{\alpha}) \\
e^{T}(\hat{\alpha}-\bar{\alpha})=0 \\
0 \leq \bar{\alpha} \leq C e \\
0 \leq \hat{\alpha} \leq C e \\
\bar{\alpha}^{T}(A w+b e-y-\varepsilon e-\bar{\xi})=0 \\
\hat{\alpha}^{T}(A w+b e-y+\varepsilon e+\hat{\xi})=0
\end{array}\right.
\end{gathered}
$$

We should proof $(\tilde{w}, \widetilde{b}, \widetilde{\bar{\xi}}, \tilde{\hat{\xi}})$ satisfy the KKT conditions

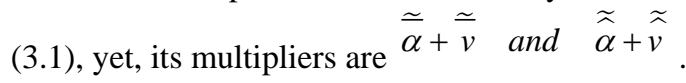




$$
\begin{aligned}
& \text { Since } A^{T}(\tilde{\hat{v}}-\tilde{\bar{v}})=0, e^{T}(\tilde{\hat{v}}-\tilde{\bar{v}})=-\tilde{b}
\end{aligned}
$$

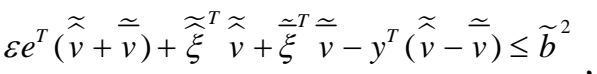

we have following formula:

$$
\begin{gathered}
A^{T}((\widetilde{\bar{\alpha}}+\widetilde{\bar{v}})-(\widetilde{\widehat{\alpha}}+\widetilde{\hat{v}}))=A^{T}(\widetilde{\bar{\alpha}}-\widetilde{\widehat{\alpha}})+A^{T}(\tilde{\bar{v}}-\widetilde{\hat{v}})=\tilde{w}+0=\tilde{w} \\
e^{T}((\widetilde{\bar{\alpha}}+\tilde{\bar{v}})-(\widetilde{\widehat{\alpha}}+\widetilde{\hat{v}}))=e^{T}(\widetilde{\bar{\alpha}}-\widetilde{\widehat{\alpha}})+e^{T}(\widetilde{\bar{v}}-\tilde{\hat{v}})=\tilde{b}-\tilde{b}=0 \\
\text { Via } 0 \leq \widetilde{\bar{\alpha}} \leq C e \text { and } 0 \leq \widetilde{\bar{v}} \leq\left(e^{T} \widetilde{\bar{v}}\right) e
\end{gathered}
$$

we have: $0 \leq \widetilde{\bar{\alpha}}+\tilde{\bar{v}} \leq\left(C+e^{T} \tilde{\bar{v}}\right) e \leq\left(C+\max \left\{e^{T} \tilde{\bar{v}}, e^{T} \tilde{\hat{v}}\right\}\right) e$ Via $0 \leq \widetilde{\hat{\alpha}} \leq C e$ and $0 \leq \tilde{\hat{v}} \leq\left(e^{T} \tilde{\hat{v}}\right) e$, we have:

$$
0 \leq \widetilde{\hat{\alpha}}+\tilde{\hat{v}} \leq\left(C+e^{T} \tilde{\hat{v}}\right) e \leq\left(C+\max \left\{e^{T} \tilde{\bar{v}}, e^{T} \tilde{\hat{v}}\right\}\right) e
$$

Since $\tilde{\bar{v}} \geq 0$,

$$
A \tilde{w}+\tilde{b} e-y-\varepsilon e-\tilde{\tilde{\xi}} \leq 0
$$

we have:

$$
\begin{aligned}
& (\widetilde{\bar{\alpha}}+\widetilde{\bar{v}})^{T}(A \tilde{w}+\tilde{b} e-y-\varepsilon e-\tilde{\bar{\xi}}) \\
& =\widetilde{\bar{\alpha}}^{T}(A \tilde{w}+\widetilde{b} e-y-\varepsilon e-\tilde{\bar{\xi}})+\stackrel{\sim}{\sim}(A \tilde{w}+\widetilde{b} e-y-\varepsilon e-\tilde{\bar{\xi}}) \quad \text { With } \\
& =\stackrel{\sim}{v} T^{T}(A \tilde{w}+\tilde{b} e-y-\varepsilon e-\widetilde{\widetilde{\xi}}) \leq 0
\end{aligned}
$$$$
\text { the same reason, from } \widetilde{\widehat{v}} \geq 0 \text { and } A \tilde{w}+\widetilde{b} e-y+\varepsilon e+\widetilde{\widetilde{\xi}} \geq 0 \text {, }
$$
we have:

$$
\begin{aligned}
& (\widetilde{\hat{\alpha}}+\widetilde{\widehat{v}})^{T}(A \tilde{w}+\widetilde{b} e-y+\varepsilon e+\widetilde{\widehat{\xi}}) \\
= & \widetilde{\widehat{\alpha}}^{T}(A \tilde{w}+\widetilde{b} e-y+\varepsilon e+\widetilde{\widehat{\xi}})+\widetilde{\widehat{v}}^{T}(A \widetilde{w}+\widetilde{b} e-y+\varepsilon e+\widetilde{\widehat{\xi}}) \\
= & \widetilde{\widehat{v}}^{T}(A \widetilde{w}+\widetilde{b} e-y+\varepsilon e+\widetilde{\widetilde{\xi}}) \geq 0
\end{aligned}
$$

The following section is to validate

$$
\begin{gathered}
{\widetilde{\sim^{T}}}^{T}(A \tilde{w}+\tilde{b} e-y-\varepsilon e-\widetilde{\bar{\xi}}) \geq 0 \\
\text { and } \widetilde{\widetilde{v}}^{T}(A \tilde{w}+\widetilde{b} e-y+\varepsilon e+\widetilde{\widetilde{\xi}}) \leq 0 .
\end{gathered}
$$
have:

$$
\begin{aligned}
& \widetilde{\widehat{v}}^{T}(A \widetilde{w}+\widetilde{b} e-y+\varepsilon e+\widetilde{\widehat{\xi}})-\widetilde{\sim}^{T}(A \widetilde{w}+\widetilde{b} e-y-\varepsilon e-\widetilde{\widetilde{\xi}}) \\
& =\tilde{w}^{T} A^{T} \widetilde{\widehat{v}}+\tilde{b} e^{T} \widetilde{\widehat{v}}-y^{T} \widetilde{\widehat{v}}+\varepsilon e^{T} \widetilde{\widehat{v}}+\widetilde{\widehat{\xi}}^{T} \widetilde{\widehat{v}}-\widetilde{w}^{T} A^{T} \widetilde{\widetilde{v}}-\widetilde{b} e^{T} \widetilde{\widetilde{v}}
\end{aligned}
$$

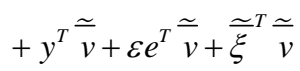

$$
\begin{aligned}
& =\tilde{w}^{T}\left(A^{T}(\widetilde{\hat{v}}-\widetilde{\bar{v}})\right)+\widetilde{b}\left(e^{T}(\widetilde{\widehat{v}}-\widetilde{\bar{v}})\right)-y^{T}(\widetilde{\widehat{v}}-\widetilde{\bar{v}})+\varepsilon e^{T}(\widetilde{\hat{v}}-\widetilde{\bar{v}}) \\
& +\widetilde{\widehat{\xi}}^{T} \widetilde{\widehat{v}}+\widetilde{\bar{\xi}}^{T} \stackrel{\widetilde{v}}{ } \\
& =\varepsilon e^{T}(\widetilde{\widehat{v}}-\widetilde{\bar{v}})+\widetilde{\widehat{\xi}}^{T} \widetilde{\widehat{v}}+\tilde{\bar{\xi}}^{T} \widetilde{\underline{v}}-y^{T}(\widetilde{\widehat{v}}-\widetilde{\bar{v}})-\widetilde{b}^{2} \leq 0
\end{aligned}
$$$$
\text { That is to say, }
$$$$
\widetilde{\widehat{v}}^{T}(A \widetilde{w}+\widetilde{b} e-y+\varepsilon e+\widetilde{\widetilde{\xi}}) \leq \widetilde{\widetilde{v}}^{T}(A \tilde{w}+\widetilde{b} e-y-\varepsilon e-\widetilde{\widetilde{\xi}})
$$$$
\text { Since } \quad \widetilde{\hat{v}}^{T}(A \tilde{w}+\tilde{b} e-y+\varepsilon e+\widetilde{\widetilde{\xi}}) \geq 0 \text {, we have }
$$$$
\stackrel{\sim}{v}(A \widetilde{w}+\widetilde{b} e-y-\varepsilon e-\widetilde{\bar{\xi}}) \geq 0
$$$$
\text { Since } \quad \stackrel{\simeq T}{v}(A \widetilde{w}+\widetilde{b} e-y-\varepsilon e-\widetilde{\bar{\xi}}) \leq 0 \quad \text {, we have }
$$$$
\tilde{\hat{v}}^{T}(A \tilde{w}+\tilde{b} e-y+\varepsilon e+\tilde{\hat{\xi}}) \leq 0
$$$$
\text { So we have: }(\widetilde{\bar{\alpha}}+\widetilde{\bar{v}})^{T}(A \widetilde{w}+\widetilde{b} e-y-\varepsilon e-\widetilde{\bar{\xi}})=0
$$$$
\text { and }(\widetilde{\hat{\alpha}}+\widetilde{\hat{v}})^{T}(A \widetilde{w}+\widetilde{b} e-y+\varepsilon e+\widetilde{\widetilde{\xi}})=0 \text { From (1) to }
$$

we can conclude that $(\tilde{w}, \widetilde{b}, \widetilde{\xi}, \widetilde{\xi})$ is the KKT point of (2.1). (2.1) is a convex quadratic programming problem, so $(\widetilde{w}, \widetilde{b}, \widetilde{\xi}, \widetilde{\xi})$ is the Solution of (2.1). In other word, the solution of (2.2) is the solution of (2.1).

\section{CONCLUSION}

This paper bases on the form of improved problem and primal problem, relates with the relation of solutions between improved problem and primal problem of SVM, studies the property of solutions between improved problem and primal problem of SVR, and proves this property strictly. Which provides the SVR with theory basis.

\section{ACKNOWLEDGMENT}

This work is partially supported by Sub-project of National Natural Science Foundation of China(KB159141), China Scholarship Council (201406905004), Shanghai International Studies University planning project (KX171309), Shanghai International Studies University curriculum construction project (KCXJ20140203).

\section{REFERENCES}

[1] V. Vapnik. Statitical Learning Theory[M]. New York:Wiley,1998.

[2] O.L.Mangasarian,D.R.Musicant.successive overrelaxation for support vector Machines[J].IEEE Trans. On Neural Networks,1999,10(5):10321037. 
[3] Yong Quan,Jie Yang,Lixiu Yao,Chenzhou Ye.Successive OverRelaxation for Support Vector Regression[J]. Journal of Software,2004, 15(2):200-206

[4] O.L. Mangasarian,D.R. Musicant. Active Support Vector Machines classification[J]. Advances in Neural Information Processing Systems(NIPS 2000),2000.

[5] D.R.Musicant, Alexander Feinberg. Active Set Support Vector Regression[J].IEEE Transactions on Neural Networks,March 2004,Vol.15,No.2.

[6] O.L. Mangasarian L, D.R. Musicant. Langrangian Support Vector Machines[J]. Journal of Machine Learning Research 1,March 2001,161177.

[7] Xiaojian Shao, Guoping He. Lagrange Support Vector Regression[J]. Intelligent Information Management Systems and Technologies, 2005,1(3):434-440.

[8] Y.J.Lee, O. L.Mangasarian. SSVM:A Smooth Support Vector Machines for Classification[J].Computational Optimazation and Applications, 2000,20(1):5-22.

[9] Y.J.Lee,W.F. Hsieh,C.M. Huang. $\varepsilon$-SSVR:A Smooth Support Vector Machines for $\varepsilon$-insensitive Regression [J]. IEEE Transactions on Knowledge and Data Engineering, 2005,17(5):678-685.

[10] Meiqin Pan, Guoping He,etc. Improved Support Vector Machine based on Successive Overrelaxation, Chinese Journal Electronic,2006,15(4A): 822-825.

[11] Meiqin Pan, Guoping He, Xiangrong Wang.Improved Generalized Support Vector Machine based on SOR. Journal of Computational Informationystems,2008,4(6):2913-2922. 